\title{
Carbon monoxide bands in M dwarfs
}

\author{
Y. V. Pavlenko ${ }^{1}$ and H. R. A. Jones ${ }^{2}$ \\ 1 Main Astronomical Observatory of Academy of Sciences of Ukraine, Golosiiv woods, Kyiv-127, 03680 Ukraine \\ e-mail: yp@mao.kiev.ua \\ 2 Astrophysics Research Institute, Liverpool John Moores University, Egerton Wharf, Birkenhead CH41 1LD, UK \\ e-mail: hraj@astro.livjm.ac.uk
}

Received 18 July 2002 / Accepted 30 September 2002

\begin{abstract}
We compare the observational and theoretical spectra of the $\Delta v=2 \mathrm{CO}$ bands in a range of $\mathrm{M}$ dwarfs. We investigate the dependence of theoretical spectra on effective temperatures as well as carbon abundance. In general we find that the synthetic $\mathrm{CO}$ bands fit the observed data extremely well and are excellent diagnostics. In particular the synthetic spectra reasonably match observations and the best fit temperatures are similar to those found by empirical methods. We also examine the ${ }^{12} \mathrm{C} /{ }^{13} \mathrm{C}$ isotopic ratio. We find that fundamental ${ }^{13} \mathrm{CO}$ bands around 2.345 and $2.375 \mu \mathrm{m}$ are good discriminators for the ${ }^{12} \mathrm{C} /{ }^{13} \mathrm{C}$ ratio in $\mathrm{M}$ dwarfs. The $2.375 \mu \mathrm{m}$ band is more useful because it doesn't suffer such serious contamination by water vapour transitions. Our current dataset does not quite have the wavelength coverage to perform a reliable determination of the ${ }^{12} \mathrm{C} /{ }^{13} \mathrm{C}$ ratio in $\mathrm{M}$ dwarfs. For this we recommend observing the region 2.31-2.40 $\mu \mathrm{m}$ at a resolution of better than 1000. Alternatively the observational problems of contamination by water vapour at $2.345 \mu \mathrm{m}$ maybe solved by observing at resolutions of around 50000 . We also investigated the possibility of using the $\Delta v=1 \mathrm{CO}$ bands around $4.5 \mu \mathrm{m}$. We find that the contamination due to water vapour is even more of a problem at these wavelengths.
\end{abstract}

Key words. stars: fundamental parameter

\section{Introduction}

More than $70 \%$ of stars in the vicinity of the Sun are M dwarfs. These numerous low-mass stars $\left(0.08 M_{\odot} \leq M<0.6 M_{\odot}\right)$, together with substellar objects - brown dwarfs $\left(M \leq 0.08 M_{\odot}\right)$ can contain an appreciable amount of the baryonic matter in the Galaxy. Research of M dwarf spectra are of interest for many branches of modern astrophysics. Verification of the theory of stellar evolution and structure of stars, the detection among $\mathrm{M}$ dwarfs of a subset of young brown dwarfs, the physical state of plasma of their atmospheres at low temperatures, as well as the chemical and physical processes of dust formation are only a few of them.

The dominant opacity sources in the optical and IR spectra of $\mathrm{M}$ dwarfs is absorption by band systems of diatomic molecules, such as $\mathrm{TiO}$ and $\mathrm{VO}$, as well as rotationalvibrational bands of $\mathrm{H}_{2} \mathrm{O}$. M dwarf infrared spectra additionally contain absorption bands of $\mathrm{CO}$. Apart from being readily identifiable in $\mathrm{M}$ dwarf spectra, $\mathrm{CO}$ bands have the advantage of being extremely well modelled. This is in contrast to the relatively poor modelling of other molecules and atomic lines in $\mathrm{M}$ dwarf atmospheres. For example, the structure of rotationalvibrational levels of two-nuclear molecules $\mathrm{CO}$ is much simpler, than $\mathrm{H}_{2} \mathrm{O}$. That allows computations of $\mathrm{CO}$ line lists to

Send offprint requests to: Ya. V. Pavlenko, e-mail: yp@mao.kiev.ua be carried out with high accuracy (e.g., Goorvitch 1994). One of the most promising observational regions is located in the $K$ band from 2.2 to $2.4 \mu \mathrm{m}$. Second overtone bands ${ }^{12} \mathrm{C}^{16} \mathrm{O}$ and ${ }^{13} \mathrm{C}^{16} \mathrm{O}$ are located here. As well as parameters such as effective temperature and gravity, they can be used for determination of carbon and/or oxygen abundances and the ${ }^{12} \mathrm{C} /{ }^{13} \mathrm{C}$ ratio in atmospheres of late-type stars. The determination of the ${ }^{12} \mathrm{C} /{ }^{13} \mathrm{C}$ ratio in $\mathrm{M}$ dwarf atmospheres is especially interesting. Following the conventional theory of stellar evolution (see Aller \& McLaughlin 1965), M dwarfs save their initial ${ }^{12} \mathrm{C} /{ }^{13} \mathrm{C}$ from their time of formation. Since the galactic ${ }^{12} \mathrm{C} /{ }^{13} \mathrm{C}$ ratio is expected to change by around a factor of four over the lifetime of our Galaxy, the determination of the ${ }^{12} \mathrm{C} /{ }^{13} \mathrm{C}$ ratio for $\mathrm{M}$ dwarfs potentially gives a strong constraint on their age. However, determining the $12 \mathrm{C} / 13 \mathrm{C}$ ratio is only a strong constraint on age if that ratio is a single-valued function of time and Galactic location. If that underlying assumption is not valid, any dispersion of ${ }^{12} \mathrm{C} /{ }^{13} \mathrm{C}$ in M-dwarf atmospheres might give for us some clues about mixing processes inside our Galaxy. Until now, measurements of the ${ }^{12} \mathrm{C} /{ }^{13} \mathrm{C}$ ratio for planetary nebulae, red giants and asymptotic giant branch stars do not fit particularly well with theory. Nonetheless, the discrepancies found for such evolved stars may well arise from the lack of realistic stellar models. Atmospheric models need to include additional physical processes in their prescription for mixing between nucleosynthetic cores and observable 
atmospheres (Palla et al. 2000). M dwarfs, on the other hand, are not expected to modify their ${ }^{12} \mathrm{C} /{ }^{13} \mathrm{C}$ and are fully convective. Thus the ${ }^{12} \mathrm{C} /{ }^{13} \mathrm{C}$ ratios for a diverse sample of $\mathrm{M}$ dwarfs is expected to be a relatively straight-forward function of time and galactic location.

Until now studies of $\mathrm{CO}$ bands in the $K$-band were mainly used for the quantitative analysis of the evolution of ${ }^{12} \mathrm{C} /{ }^{13} \mathrm{C}$ in atmospheres of red giants (cf. Hinkle 1978; Sneden \& Pilachowski 1984; McGregor 1987; Lazaro et al. 1991; Suntzeff \& Smith 1992; Scott et al. 1994; Pilachovski et al. 1997). However, they are also observed in the spectra of many objects - from symbiotic stars (Schild et al. 1992), Be stars (Kraus et al. 2000) and supernova remnants (Gerardy et al. 2000) up to planets (Forbes et al. 1970). Baldwin et al. (1973) and many others since have shown the prevalence of the $\mathrm{CO}$ bands in the $K$ band spectra of M dwarfs. Viti et al. (2002) have shown that the $\mathrm{CO}$ bands in the $K$ band are relatively well-matched by synthetic spectra and thus can be used to determine $\mathrm{M}$ dwarf properties. In our study we make comparisons of synthetic and observed spectra across the 2.3-2.4 $\mu \mathrm{m}$ region to quantify the usefulness of the $\mathrm{CO}$ bands as a diagnostic of effective temperatures and metallicities for $\mathrm{M}$ dwarfs.

\section{Observations}

The properties of observed stars for which spectra are fitted in our paper are listed in Table 1. Observations were made with the Cooled Grating Spectrometer 4 on the UK Infrared Telescope (UKIRT) on Mauna Kea, Hawaii. The instrument then had a $58 \times 62 \mathrm{InSb}$ array which was moved in the focal plane in order to three times over-sample the spectrum. Sky subtraction was performed by nodding the telescope approximately 30 arcsec up and down the slit, ensuring that during alternate "object" and "sky" observations the star remained on the detector. The observations presented in this paper were made during three nights, 1992 May 8, 10 and 12 in reasonable optical seeing $(0.75-1.5$ arcsec) and of atmospheric humidity (10-50 per cent). The airmass difference between object and standard never exceeded 0.05 and so we are confident that the spectra have good cancellation of atmospheric features.

The 150 lines $\mathrm{mm}^{-1}$ grating was used in third order with the $150 \mathrm{~mm}$ focal length camera at a central grating wavelength of $2.34 \mu \mathrm{m}$. This grating position was chosen to be closed to the centre of the $\mathrm{CO}$ bands, at a maximum in the grating efficiency have high atmospheric transmission ( $>95$ per cent) and gives a relatively high resolution, $\lambda / \Delta \lambda$, where $\Delta \lambda$ is the detector resolution at wavelength $\lambda$, of 1085 (equivalent to $276 \mathrm{~km} \mathrm{~s}^{-1}$ ).

\subsection{Standards}

Stars in the spectral type ranges B5-A5 and F6-G0 were used to remove the effects of atmospheric absorption. These standards were expected to be featureless at the spectral resolution used and to be well described by a Rayleigh-Jeans tail. However, the F6-G0 standards were not very useful as they show weak metal lines. Although the lines tended to be weaker and different from the $\mathrm{M}$ dwarf features we avoided using them where possible. In the reduction of spectra, we have followed the same procedures as in Jones et al. (1996). All observations were wavelength calibrated using lines from observations of an argon lamp in the CGS4 calibration unit. This procedure is typically accurate to $0.1 \Delta \lambda$.

\section{Procedure}

We used local thermal equilibrium (LTE) model atmospheres of M dwarfs with effective temperatures $T_{\text {eff }}=2400-3800 \mathrm{~K}$ from the NextGen grid of Hauschildt et al. (1999). Unless otherwise mentioned all models are for $\log g=5.0$ and solar metallicity. Computations of LTE synthetic spectra were carried out by the program WITA6 (Pavlenko 2000) assuming LTE, hydrostatic equilibrium for an one-dimensional model atmosphere and without sources and sinks of energy. The equations of ionisation-dissociation equilibrium were solved for media consisting of atoms, ions and molecules. We took into account $\sim 100$ components (Pavlenko 2000). The constants for equations of chemical balance were taken from Tsuji (1973). We adopt the dissociation potential $D_{0}=9.5 \mathrm{eV}$ for $\mathrm{H}_{2} \mathrm{O}$ and $D_{0}=11.105 \mathrm{eV}$ for ${ }^{12} \mathrm{C}^{16} \mathrm{O},{ }^{13} \mathrm{C}^{16} \mathrm{O}$. It is worth noting that the chemical balance in $\mathrm{M}$ dwarf atmospheres is governed by the CO molecule (see Sect. 4.1).

Line lists of ${ }^{1} \mathrm{H}_{2}^{16} \mathrm{O}$ were computed using the AMES database (Partrige \& Schwenke 1998). The partition functions of $\mathrm{H}_{2} \mathrm{O}$ were also computed on these data (Pavlenko 2002). We used ${ }^{12} \mathrm{C}^{16} \mathrm{O}$ and ${ }^{13} \mathrm{C}^{16} \mathrm{O}$ line lists of Goorvitch (1994). The CO partition functions were taken from Gurvitz et al. (1989). The atomic line list was taken from VALD (Kupka et al. 1999).

The profiles of molecular and atomic lines are determined using the Voigt function $H(a, v)$, parameters of their natural broadening $C_{2}$ and van der Waals broadening $C_{4}$ from databases (Kupka et al. 1999) or in their absence computed following Unsold (1955). Owing to the low temperatures in M dwarf atmospheres and consequently, electron densities, Stark broadening may be neglected. As a whole the effects of pressure broadening prevail. Computations for synthetic spectra were carried out with a resolution of $0.00005 \mu \mathrm{m}$ for microturbulent velocity $v_{\mathrm{t}}=2 \mathrm{~km} \mathrm{~s}^{-1}$.

The instrumental broadening was modelled by triangular profiles set to the resolution of the observed spectra. To find the best fits to observed spectra we follow the scheme of Jones et al. (2002). Namely, for every spectrum we carry out the minimisation of a $3 \mathrm{D}$ function $S=f\left(x_{\mathrm{s}}, x_{\mathrm{f}}, x_{\mathrm{w}}\right)=1 / N \times$ $\sum\left(1-F_{\text {obs }} / F_{\text {synt }}\right)^{2}$, where $F_{\text {obs }}, F_{\text {synt }}$ are observed and computed fluxes, $N$ is the number of points in observed spectrum to be fitted, $x_{\mathrm{s}}, x_{\mathrm{f}}, x_{\mathrm{w}}$ are relative shift in wavelength scale, a normalisation factor which was used to coincide observed and computed spectra and parameter of instrumental broadening, respectively.

\section{Results}

\section{1. $\mathrm{CO}$ and $\mathrm{H}_{2} \mathrm{O}$ molecules in $\mathrm{M}$ dwarf atmospheres}

The overall chemical balance in the atmospheres of cool stars depends significantly on the $\mathrm{C} / \mathrm{O}=\log N(\mathrm{C})-\log N(\mathrm{O})$ (see Tsuji 1973). In the atmospheres of $\operatorname{cool}\left(T_{\text {eff }}<3600 \mathrm{~K}\right)$ oxygen 
Table 1. The properties of observed stars are taken from Jones et al. (1996). Spectral types are taken from Kirkpatrick et al. (1991).

\begin{tabular}{cccccccc}
\hline \hline Object & $d(\mathrm{pc})$ & KIN & COL & Sp Type & $M_{\mathrm{K}}(\mathrm{CIT})$ & $V_{\mathrm{C}}-K_{\mathrm{CIT}}$ & $I_{\mathrm{CCD}}-L_{\text {MKO }}^{\prime}$ \\
\hline Gl 411 & 2.5 & OD & OD/H & dM2 & 6.34 & 4.11 & 2.24 \\
Gl 699 & 2.39 & OD/H & OD/H & dM4 & 8.20 & 5.04 & 2.59 \\
Gl 406 & 1.83 & OD & - & dM6 & 9.19 & 7.37 & 3.70 \\
VB 10 & 5.79 & OD & - & dM8 & 9.99 & 8.70 & 4.65 \\
LHS 2924 & 10.72 & OD & - & dM9 & 10.52 & 8.91 & 5.19 \\
\hline
\end{tabular}

rich stars $\mathrm{C} / \mathrm{O}<0$, almost all atoms of carbon are bound in $\mathrm{CO}$ molecules. $\mathrm{CO}$ and $\mathrm{H}_{2} \mathrm{O}$ are the most abundant molecules containing oxygen atoms.

In the atmospheres of $\mathrm{M}$ dwarfs with $T_{\text {eff }}>2600 \mathrm{~K}$, molecules of $\mathrm{CO}$ dominate by number in comparison with other oxygen containing molecules (Tsuji 1973). Throughout most of the atmosphere changes of the ratio $\mathrm{C} / \mathrm{O}$ by $\pm 0.2 \mathrm{dex}$ have little effect on the molecular densities of CO (Fig. 1). In contrast the dependence of molecular densities on $\mathrm{H}_{2} \mathrm{O}$ on $\log N(\mathrm{C})$ is more complex. At lower $T_{\text {eff }}$ (e.g. Fig. 1b) the dependence of $n_{\mathrm{H}_{2} \mathrm{O}}$ on $\log N(\mathrm{C})$ in $\mathrm{M}$ dwarfs atmospheres weakens appreciably though the chemical balance remains governed by $\mathrm{CO}$. In general, $\mathrm{H}_{2} \mathrm{O}$ and $\mathrm{CO}$ are competitors for boundfree oxygen atoms. Therefore their molecular densities respond in opposite ways to changes of carbon abundance. For solar abundances of oxygen and carbon, molecular densities of $\mathrm{H}_{2} \mathrm{O}$ and $\mathrm{CO}$ are comparable. $\mathrm{CO}$ molecular densities increase only a little when $\log N(\mathrm{C})$ increases from -3.48 up to $-3.12^{1}$. At the same time $\mathrm{CO}$ reduces molecular densities of $\mathrm{H}_{2} \mathrm{O}$. However, relative changes of $\mathrm{H}_{2} \mathrm{O}$ densities are comparatively weak for variations of $\log N(\mathrm{C})$ in the range from -3.48 to -3.18 . Only when the carbon abundance rises to $\log N(\mathrm{C}) \sim$ -3.18 , molecular densities of $\mathrm{H}_{2} \mathrm{O}$ decrease, as an appreciable fraction of oxygen atoms become bound in $\mathrm{CO}$.

The relative strengths of $\mathrm{H}_{2} \mathrm{O}$ and $\mathrm{CO}$ bands at $2600 \mathrm{~K}$ and $3600 \mathrm{~K}$ are shown in Fig. 2. These spectra were computed separately by switching on/off the absorption of $\mathrm{H}_{2} \mathrm{O}$ or $\mathrm{CO}$ bands.

The relative intensity of the $\mathrm{CO}$ bands decrease from $\Delta v=1$ to $\Delta v=3$. Although the second overtone bands $(\Delta v=2)$ of $\mathrm{CO}$ have intermediate intensities they are located in a relatively accessible region for observation.

\subsubsection{Bands of the second overtone of $\mathrm{CO}$ from $2.28-2.4 \mu \mathrm{m}$}

The relative intensities of ${ }^{12} \mathrm{C}^{16} \mathrm{O}{ }^{13} \mathrm{C}^{16} \mathrm{O}$ and $\mathrm{H}_{2} \mathrm{O}$ bands from 2.28-2.38 $\mu \mathrm{m}$ are shown in Fig. 3. These computations are carried out for a model atmosphere with $2600 \mathrm{~K}$ and ${ }^{12} \mathrm{C} /{ }^{13} \mathrm{C}=0.5$. The large ratio of ${ }^{12} \mathrm{C} /{ }^{13} \mathrm{C}$ was chosen to show the positions of the main details which might be observed in $\mathrm{M}$ dwarf spectra. Overall, $\mathrm{H}_{2} \mathrm{O}$ absorption dominates the infrared spectral region. However, strong features are also formed there by first overtone bands of ${ }^{12} \mathrm{C}^{16} \mathrm{O}$. The positions of the ${ }^{13} \mathrm{C}^{16} \mathrm{O}$ bandheads are marked by vertical arrows in Fig. 3. The results of our ${ }^{13} \mathrm{C}^{16} \mathrm{O}$ modelling of spectra of our dwarfs are discussed in Sects. 4.1.3 and 4.1.4.

\footnotetext{
${ }_{1}$ In our paper we use an abundance scale $\Sigma N_{i}=1$.
}
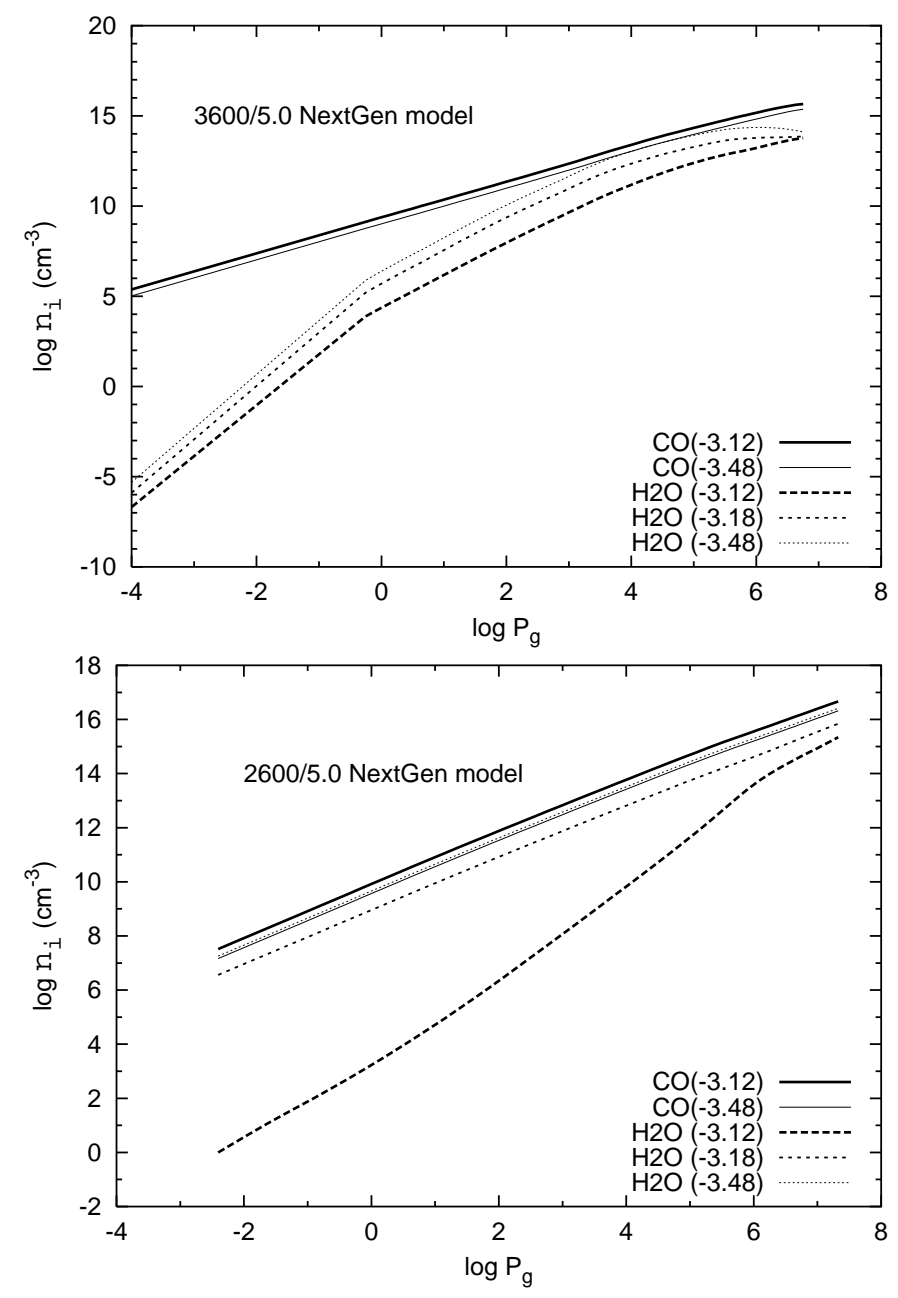

Fig. 1. Molecular densities of $\mathrm{H}_{2} \mathrm{O}$ and $\mathrm{CO}$ in atmospheres of M dwarfs 3600/5.0 and 2600/5.0 as a function of gas pressure $P_{\mathrm{g}}$. The case of $\log N(\mathrm{C})=-3.48$ and $\log N(\mathrm{O})=-3.12$ represent the solar abundances case.

\subsubsection{Fits to the observed spectra}

Our numerical procedure for the determination of best fits allows us to quantify the possible solutions. For each observed spectra we compute synthetic spectra for different $T_{\text {eff }}$. We also allow for small variations of carbon abundances. Based on Fig. 1, we suggest that small variations of $\log N(\mathrm{C})$ from -3.48 to -3.28 cannot substantially affect the temperature structure of the model atmospheres. From the family of curves $S=f\left(T_{\mathrm{eff}}\right.$, $\log N(\mathrm{C})$ ) we choose "the best solution" for each observed spectrum. 


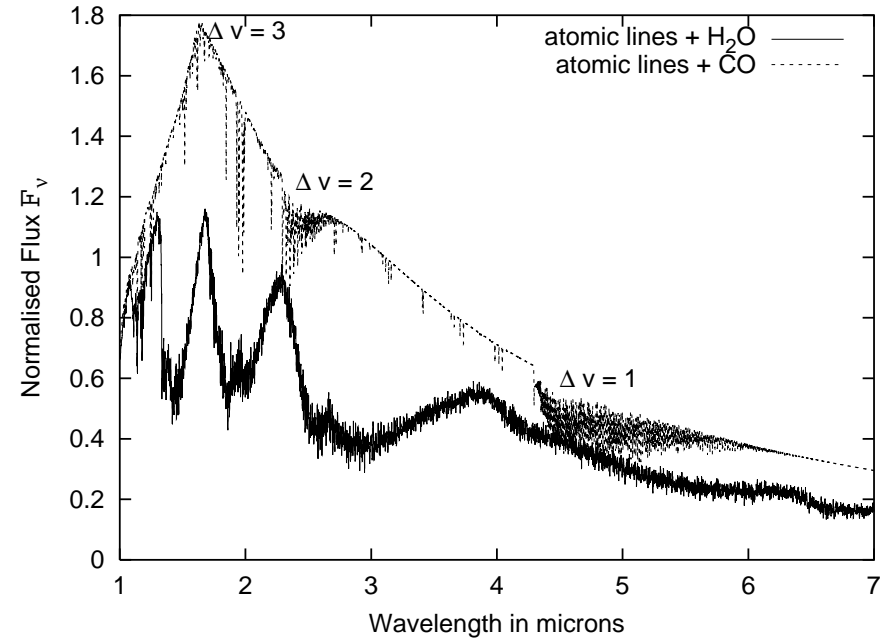

Fig. 2. The relative strength of ${ }^{12} \mathrm{C}^{16} \mathrm{O}$ and $\mathrm{H}_{2} \mathrm{O}$ bands in a $2600 \mathrm{~K}$ synthetic spectrum. The positions and relative strengths of the $\mathrm{CO}$ bands can be seen.

LHS2924. The observed spectrum of this M9V object is of relatively low signal-to-noise. For example, our best fit minimisation value $S_{\min }$ for LHS2924 is a factor 10 larger than VB10 (Fig. 4). Our best fit provides $T_{\text {eff }}=2800 \mathrm{~K}$ for $\log N(\mathrm{C})=-3.28$ and $T_{\mathrm{eff}}=2600 \mathrm{~K}$ for a solar abundance of carbon $(\log N(\mathrm{C})=-3.48)$.

VB10. VB10 is an archetypal late-type M dwarf with an effective temperature $T_{\text {eff }} \sim 2600 \mathrm{~K}$ (Jones et al. 1996). Lithium lines are absent from VB10's spectrum (Schweitzer et al. 1996; Martin 1999) and it has typical old disk properties (Leggett 1992) and hence VB10 is a star right at the end of the main sequence. For the solar abundance of carbon and other elements $S_{\min }$ occurs for $2600 \mathrm{~K}$. However, if we increase $\log N(\mathrm{C}), S$ decreases, and we find a best fit value of $2800 \mathrm{~K}$ for $\log N(\mathrm{C})=-3.28 \pm 0.1$. In general, for higher $\log N(\mathrm{C})$, CO bands intensities increase and best fits move to higher $T_{\text {eff. }}$. The same effect is found for all dwarfs of our sample.

GJ406. Best fits are found for $2800 \mathrm{~K}$ for the solar abundance case, and $3000 \mathrm{~K}$ for $\log N(\mathrm{C})=-3.28$ (Fig. 6). Our new estimation of effective temperature corresponds better with empirical values for the effective temperature of the spectral class M6V than the Jones et al. (2002) analysis found using a similar technique but using a region dominated by water vapour. Our best fits for GJ406 are found for solar metallicity rather than the metal poor result found by Jones et al. (2002).

GJ699. The observed spectrum of GJ699 (M4V) is of lower resolution and taken from the setup used in Jones et al. (1994). The details in observed spectrum are best fit by metalpoor $(\log N(\mathrm{C})=-3.68$; i.e., $[\mathrm{C}]=-0.2)$ synthetic spectra of $T_{\text {eff }}=3200 \mathrm{~K}$ (see Fig. 7). The same best fit temperature is also found for the solar abundances $\log N(\mathrm{C})=-3.48,([\mathrm{C}]=0)$ case. However, a carbon-deficient case $\log N(\mathrm{C})=-3.68$ $([C]=-0.2)$ provides lower $S$. We note that Jones et al. (2002) found an effective temperature $T_{\text {eff }}=3300 \mathrm{~K}$ and metallicity $[\mathrm{M} / \mathrm{H}]=-0.5([\mathrm{O}]=-0.5)$. On the other hand, our fits with $\log N(\mathrm{C})=-3.88([\mathrm{C}]=-0.4)$ provide less satisfactory fits. i.e. fits of higher $S$. We have probably reached the limit at
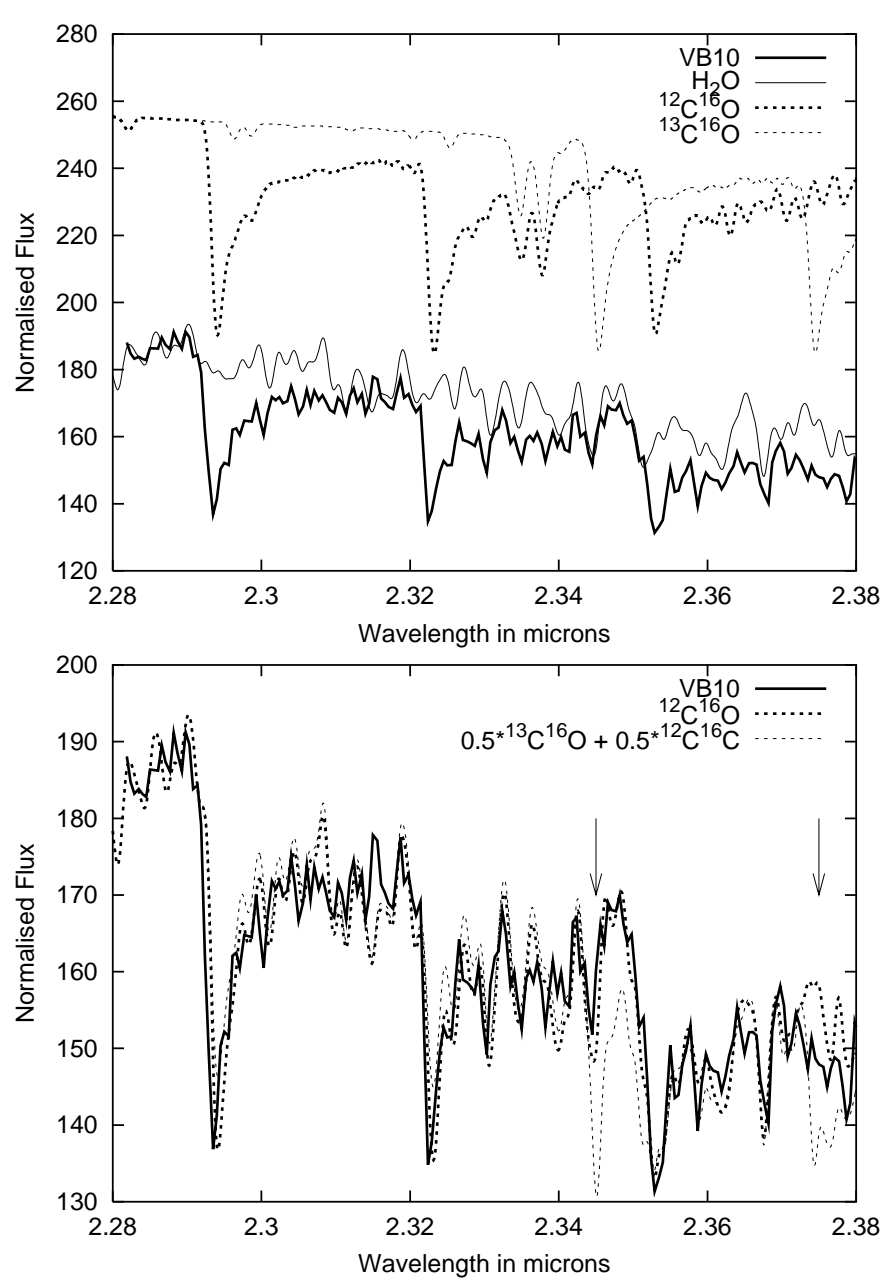

Fig. 3. The relative strength of ${ }^{12} \mathrm{C}^{16} \mathrm{O}, \mathrm{H}_{2} \mathrm{O}$ and ${ }^{13} \mathrm{C}^{16} \mathrm{O}$ bands in the spectrum of VB10 (top), and a combined spectra computed for the case of ${ }^{12} \mathrm{C}^{16} \mathrm{O}+\mathrm{H}_{2} \mathrm{O}$ and ${ }^{12} \mathrm{C}^{16} \mathrm{O}+{ }^{13} \mathrm{C}^{16} \mathrm{O}+\mathrm{H}_{2} \mathrm{O}$ absorptions. The model atmospheres shown are $2600 \mathrm{~K},{ }^{12} \mathrm{C} /{ }^{13} \mathrm{C}=0.5$ (bottom). The positions of ${ }^{13} \mathrm{C}^{16} \mathrm{O}$ bands heads are shown by arrows.

which a single metallicity parameter is appropriate for fits to the spectra of M-dwarfs.

GJ411 is the hottest dwarf in our sample. The fit to the observed spectrum is shown in Fig. 8. Within our observed spectral region the intensities of observed and computed band heads are fitted for $T_{\text {eff }}=3400 \mathrm{~K}$ and $\log N(\mathrm{C})=-3.48$ (Fig. 8). Although the relatively poorer description of the $\mathrm{M}$ dwarf spectrum slope testifies to the presence of certain problems in the definition of its physical characteristics: abundances, homogeneity of atmospheres, chromospheres or other reasons (see Sect. 5).

\subsubsection{Isotopic ratio ${ }^{12} \mathrm{C} /{ }^{13} \mathrm{C}$ in $\mathrm{M}$ dwarf atmospheres}

The temperatures in $\mathrm{M}$ dwarf interiors are relatively low $\left(T<10^{6} \mathrm{~K}\right)$, so that hydrogen burning is only possible in pp chain reactions and the hydrogen burning lifetimes are rather long (e.g. Laughlin et al. 1997). Thus despite the large convective envelopes extending down to the hydrogen burning region in a short timescales, carbon isotope abundances 
Table 2. $T_{\text {eff }}$ of our sample for solar metallicity compared with other methods: water vapour (Jones et al. 2002), infrared flux method (Tsuji et al. 1997), constant opacity (Jones et al. 1994), model fit based on temperatures derived by Leggett et al. (2000, 2001). The temperatures in italics are those derived for stars of similar spectral type.

\begin{tabular}{ccccccc}
\hline \hline Object & $\begin{array}{c}\text { Spectral } \\
\text { Type }\end{array}$ & $\begin{array}{c}T_{\text {eff }} \\
\text { This work }\end{array}$ & $\begin{array}{c}T_{\text {eff }} \\
\text { Water vapour }\end{array}$ & $\begin{array}{c}T_{\text {eff }} \\
\text { IRFM }\end{array}$ & $\begin{array}{c}T_{\text {eff }} \\
\text { Constant opacity }\end{array}$ & $\begin{array}{c}T_{\text {eff }} \\
\text { Model fit }\end{array}$ \\
\hline GJ 411 & M2V & 3400 & - & 3510 & 3471 & 3450 \\
GJ 699 & M4V & 3200 & 3300 & 3210 & 3095 & 3100 \\
GJ 406 & M6V & 2800 & 3000 & 2800 & 2670 & 2600 \\
VB10 & M8 & 2600 & - & 2250 & 2506 & 2250 \\
LHS2924 & M9 & 2600 & - & 2120 & 2219 & 2125 \\
\hline
\end{tabular}
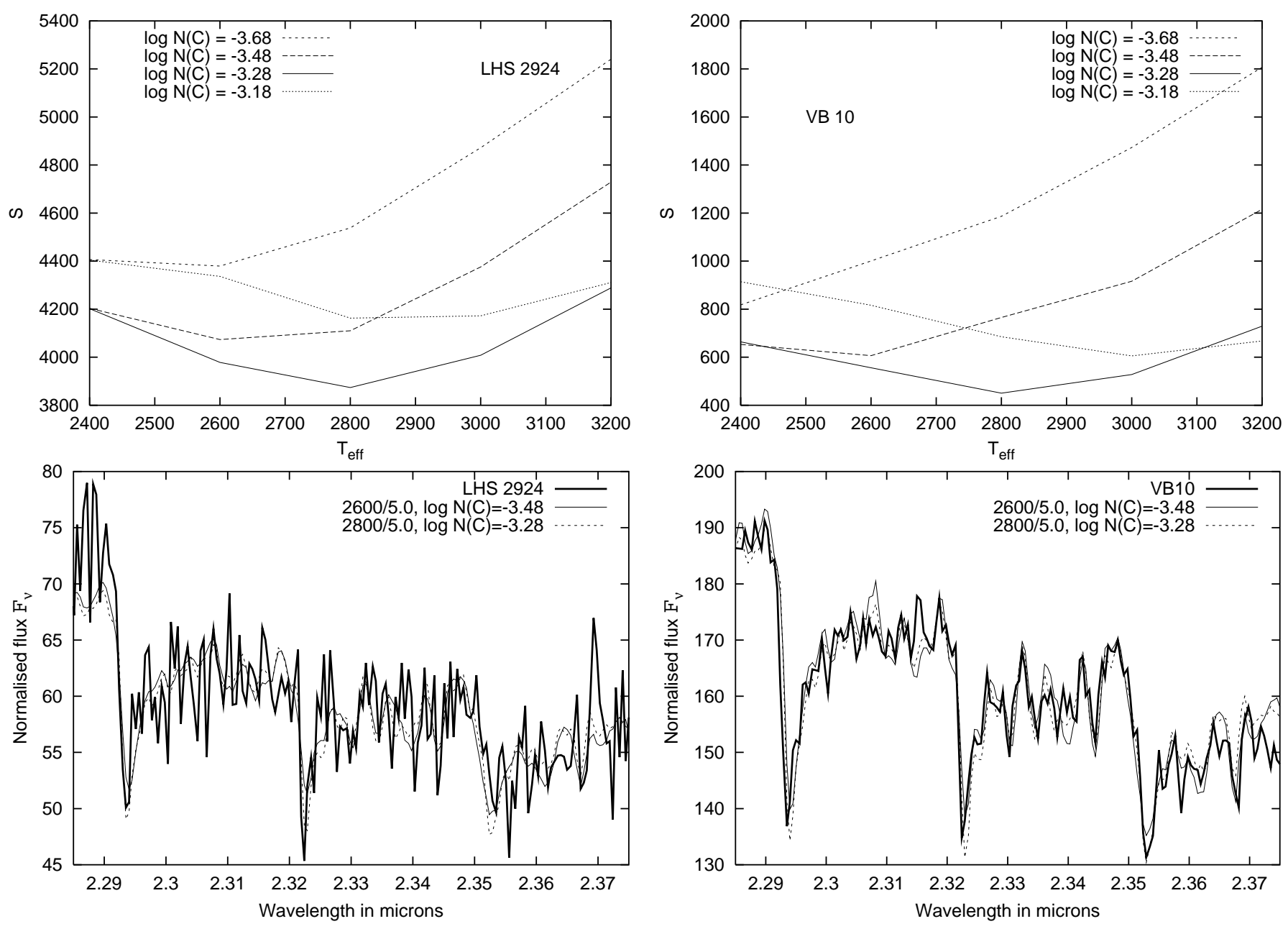

Fig. 4. Top: The dependence of $S$ on $T_{\text {eff }}$ and $\log N(\mathrm{C})$. Bottom: 2600 and $2800 \mathrm{~K}$ synthetic spectra compared to the observed LHS2924 spectrum.

cannot be changed even in Hubble-scale times. $M$ dwarfs are expected to preserve their initial carbon isotopes abundance from their time of formation. The situation is very different for massive $\left(M>0.8 M_{\odot}\right)$ stars, which burn hydrogen in CNO reactions and are observed to change their isotopic abundances of $\mathrm{C}, \mathrm{N}, \mathrm{O}$. The determination of the ${ }^{12} \mathrm{C} /{ }^{13} \mathrm{C}$ ratio in the atmospheres of $\mathrm{M}$ dwarfs might provide important constraints on the theory of the evolution of the Galaxy.

Fig. 5. Top: The dependence of $S$ on $T_{\text {eff }}$ and $\log N(\mathrm{C})$. Bottom: The best fit of synthetic spectra to the observed VB10 spectrum.

We investigated the determination of the carbon isotopic ratio in the atmospheres of our observed $\mathrm{M}$ dwarfs. The analysis of the weak ${ }^{13} \mathrm{C}^{16} \mathrm{O}$ bands in our comparatively lowresolution spectra of dwarfs is rather difficult (e.g., Fig. 9 for VB10). The $\mathrm{CO}$ band head at 2.345 is mimiced by $\mathrm{H}_{2} \mathrm{O}$ absorption. Moreover, the overall shape of the energy distribution at these wavelengths is governed by $\mathrm{H}_{2} \mathrm{O}$ absorption, Fig. 2 . From the fit of our spectra for VB10 we can estimate the lower limit ${ }^{12} \mathrm{C} /{ }^{13} \mathrm{C}>15$. For more accurate determination using this feature it will be necessary to use spectra of better 

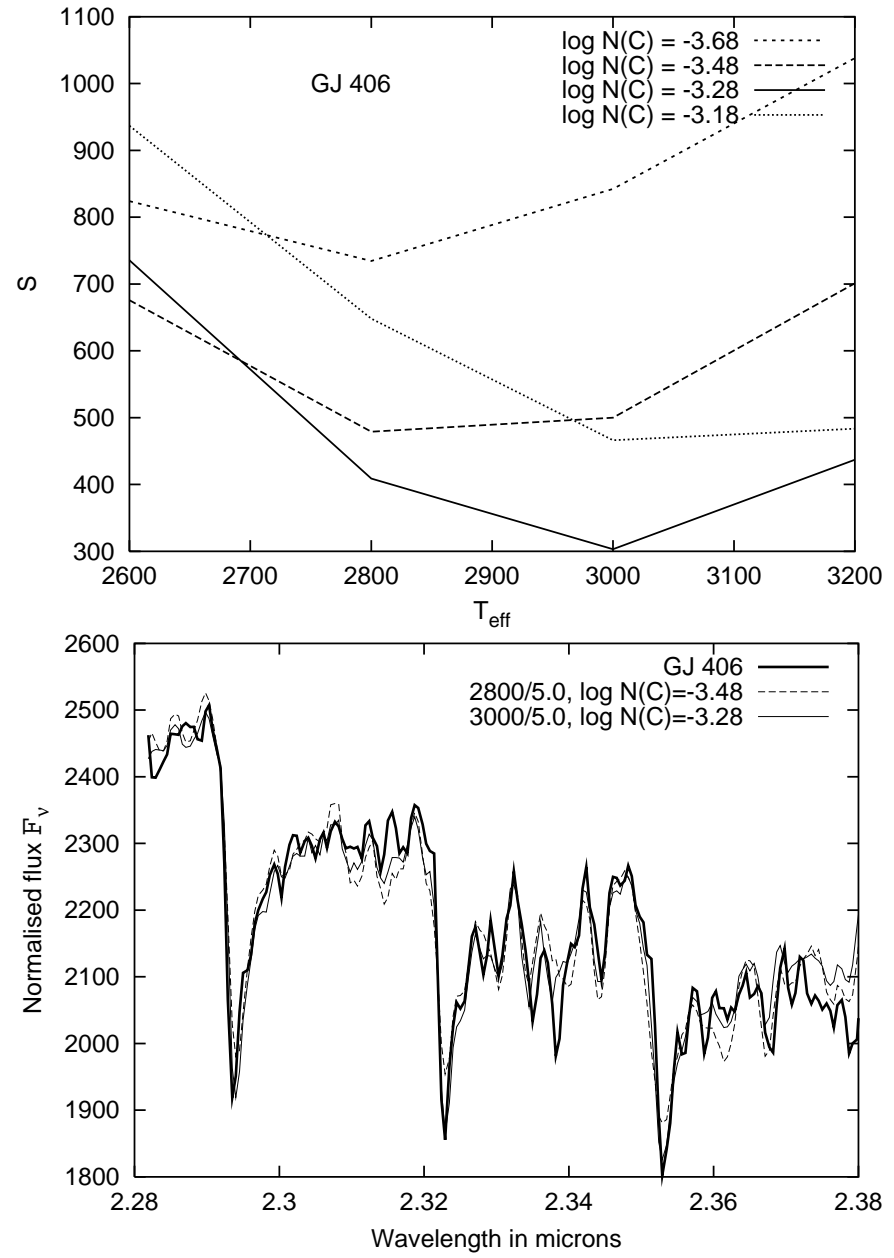

Fig. 6. The same as in Fig. 5, but for GJ406. Synthetic spectra are shown for $T_{\text {eff }}=2800$ and $3000 \mathrm{~K}$.

resolution (>50000 for ${ }^{12} \mathrm{C} /{ }^{13} \mathrm{C}$ in range 40-90, e.g., upper panel of Fig. 9). The other band of ${ }^{13} \mathrm{C}^{16} \mathrm{O}$ at $2.375 \mu \mathrm{m}$ provides a more sensitive tool for ${ }^{12} \mathrm{C} /{ }^{13} \mathrm{C}$ determination in $\mathrm{M}$ dwarf spectra. From the fit of the observed band to our models we find ${ }^{12} \mathrm{C} /{ }^{13} \mathrm{C} \sim 10$. Unfortunately our confidence in this fit is weakened because our data do not extend beyond $2.38 \mu \mathrm{m}$. To be sure we are measuring this feature we need to have data beyond $2.38 \mu \mathrm{m}$. For the other $\mathrm{M}$ dwarfs the fits are not so good so it is necessary to confirm this result with spectra of higher resolution and signal-to-noise. Nevertheless, from the fit of our spectra we can estimate the lower limit ${ }^{12} \mathrm{C} /{ }^{13} \mathrm{C}>10$ for all stars.

\subsubsection{Bands of the first overtone of $\mathrm{CO}$ from 4.3 to 4.6 microns}

As with the $\Delta v=2 \mathrm{CO}$ bands at $2.3 \mu \mathrm{m}$ it might be feasible to pursue the ${ }^{12} \mathrm{C} /{ }^{13} \mathrm{C}$ ratio using the $\Delta v=1 \mathrm{CO}$ bands at $4.4 \mu \mathrm{m}$. A theoretical picture of formation of the absorption features in the region of the bands of the first overtone of $\mathrm{CO}$ is shown in Fig. 10. Again, as for the ${ }^{13} \mathrm{CO}$ feature at $2.345 \mu \mathrm{m}$, lines of $\mathrm{H}_{2} \mathrm{O}$ dominate in region (see also Fig. 2). Bands of ${ }^{12} \mathrm{CO}$ (and ${ }^{13} \mathrm{CO}$ ) are actually of lower intensities relative to water.
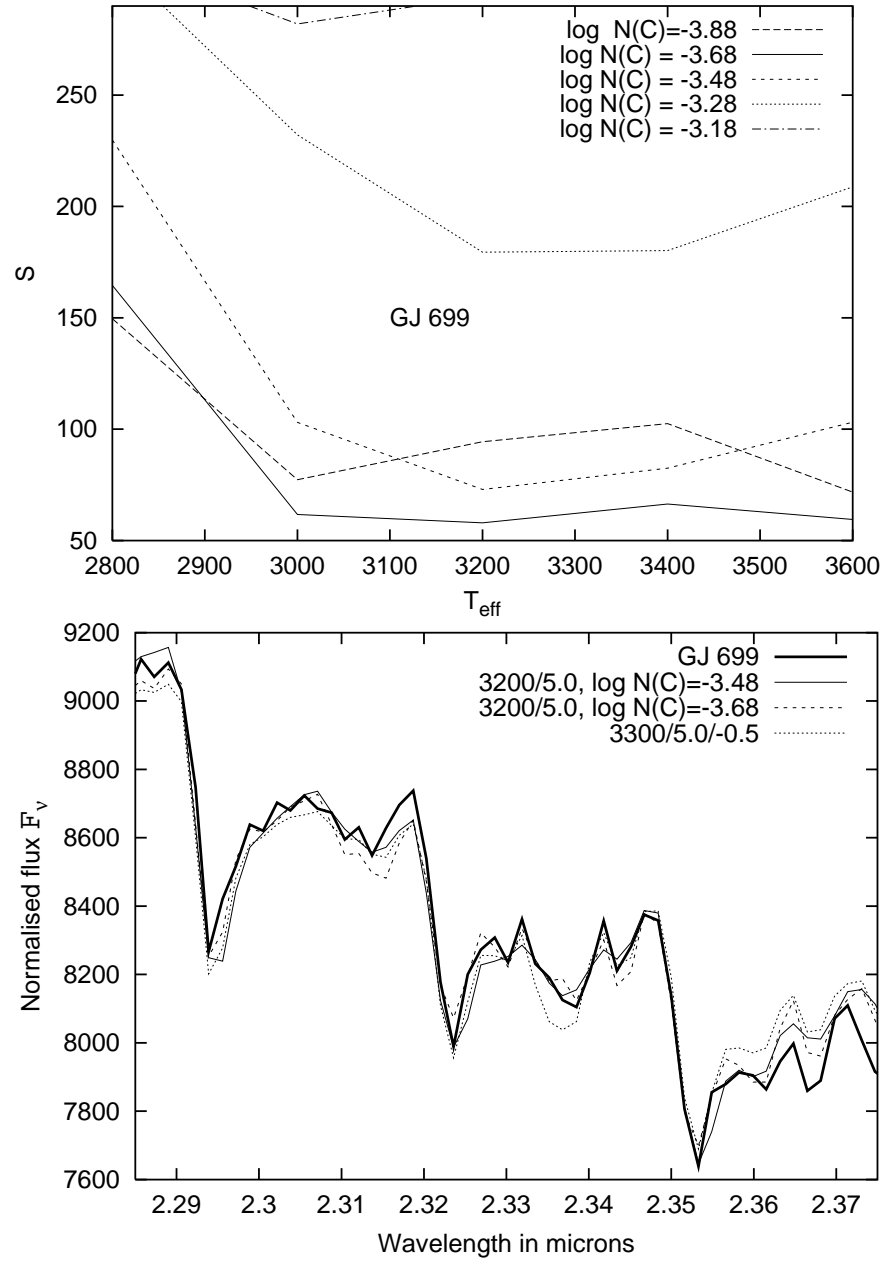

Fig. 7. The same as in Fig. 5, but for GJ699. Synthetic spectra computed for 3400 and $3600 \mathrm{~K}$ synthetic spectra are shown.

The band heads of ${ }^{13} \mathrm{C}$ fall such that their heads lie in the tails of ${ }^{12} \mathrm{C}$ bands, which are stronger. Therefore the determination of the ${ }^{12} \mathrm{C} /{ }^{13} \mathrm{C}$ ratio for $\Delta v=1 \mathrm{CO}$ bands is also problematic.

\section{Discussion}

Our effective temperature determinations are based primarily on ${ }^{12} \mathrm{C}^{16} \mathrm{O}$ lines but also affected by $\mathrm{H}_{2} \mathrm{O}$ lines. In Table 2 it can be seen that we find similar temperatures to those found for $\mathrm{M}$ dwarfs for $\mathrm{H}_{2} \mathrm{O}$ lines alone. These results support the expected quality of the ${ }^{12} \mathrm{C}^{16} \mathrm{O}$ and $\mathrm{H}_{2} \mathrm{O}$ line lists.

In general, we find $T_{\text {eff }}$ of our dwarfs with internal errors of around $\pm 150 \mathrm{~K}$. Table 2 shows $T_{\text {eff }}$ derived for $\mathrm{M}$ dwarfs by different methods. It indicates that whilst there is some agreement for the earlier type $\mathrm{M}$ dwarfs, the temperatures we derive for late spectral types are much too hot. This discrepancy arises because in the atmospheres of red dwarfs of spectral class M 6 and later (Tsuji et al. 1996; Jones \& Tsuji 1997) appreciable amounts of dust forms (e.g., Tsuji 2002) that can absorb and/or scatter radiation. This dust changes the structure of model atmospheres of $\mathrm{M}$ dwarfs, and, hence, their spectra. The outermost layers of late $\mathrm{M}$ dwarf atmospheres increase due to appearance there of the "dusty" opacity. In general, we get the 

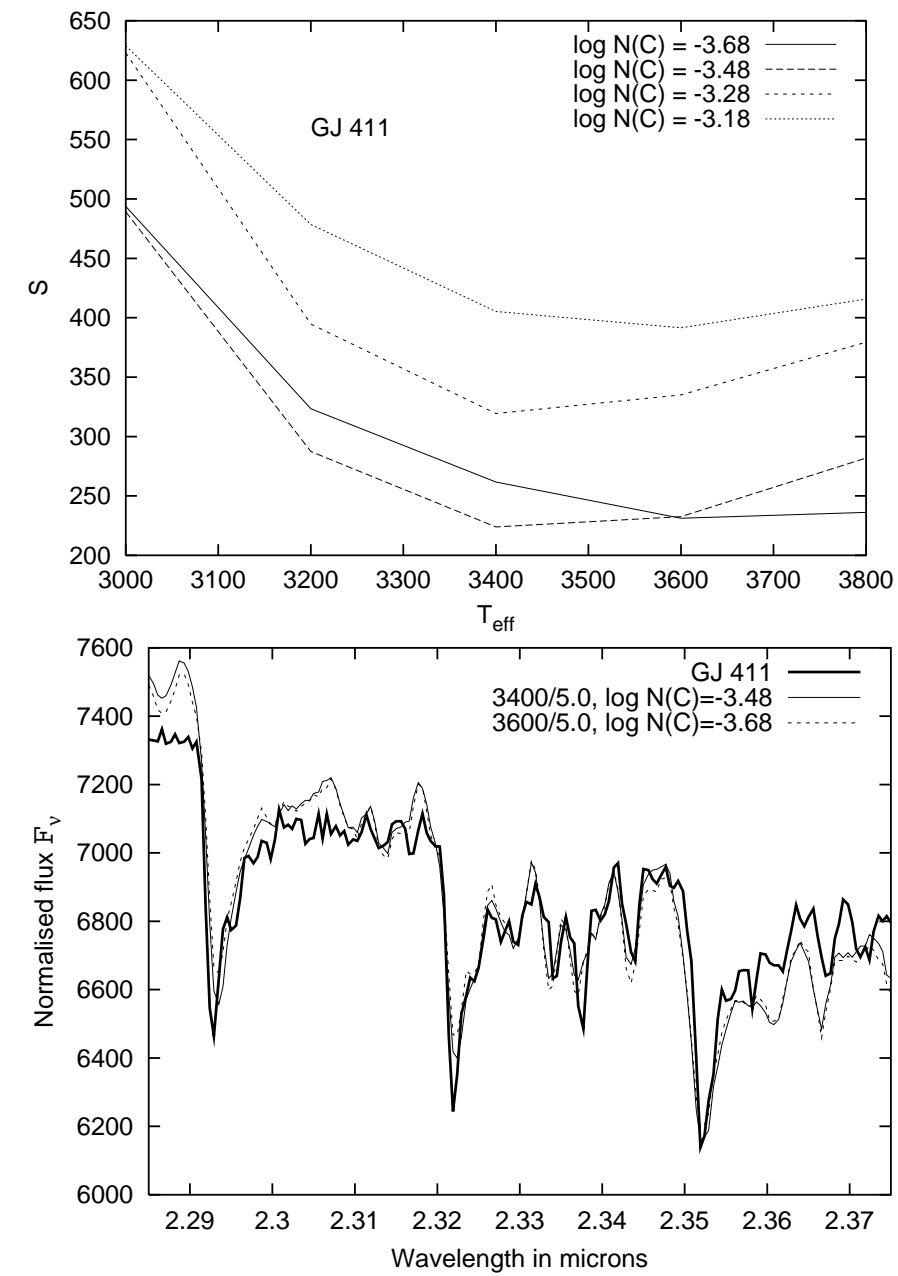

Fig. 8. The same as in Fig. 5, but for GJ411 including synthetic spectra for 3400 and $3600 \mathrm{~K}$.

much more sophisticated picture of formation of late dwarf spectra in the "dusty" atmospheres in comparison with "nondusty" case. For the case of our fits the dust in cooler stars causes the $\mathrm{CO}$ bands to be relatively weaker and thus best-fit by a high temperature, non-dusty model. Analysis of our data to determine reliable temperatures for VB 10 and LHS 2924 thus requires the use of dusty models. Such models are now in preparation (Pavlenko et al., in preparation).

NLTE (Non Local Thermal Equilibrium) effects provide another possible source of error. Carbon et al. (1976) investigated NLTE effects in CO lines of the fundamental systems formed in the atmospheres of stars of giant late-type spectral classes. However, they used a rather simple model of rotational-vibrational levels of $\mathrm{CO}$. Furthermore they considered the model atmospheres of red giants, where the densities are much lower, than in M dwarf atmospheres. Nevertheless, they showed the possibility of NLTE effects in CO lines, formed on depths $\tau_{1 \mu \mathrm{m}}<10^{-4}$. CO lines in our cases are strong, they are formed deep in the atmosphere (due to the low opacities in continuum). The formation of absorption lines of $\mathrm{CO}$ occurs in $\mathrm{M}$ dwarfs atmospheres on a background of strong $\mathrm{H}_{2} \mathrm{O}$ absorption. The additional absorption should strengthen the thermalisation of the radiation field on $\mathrm{CO}$ lines.
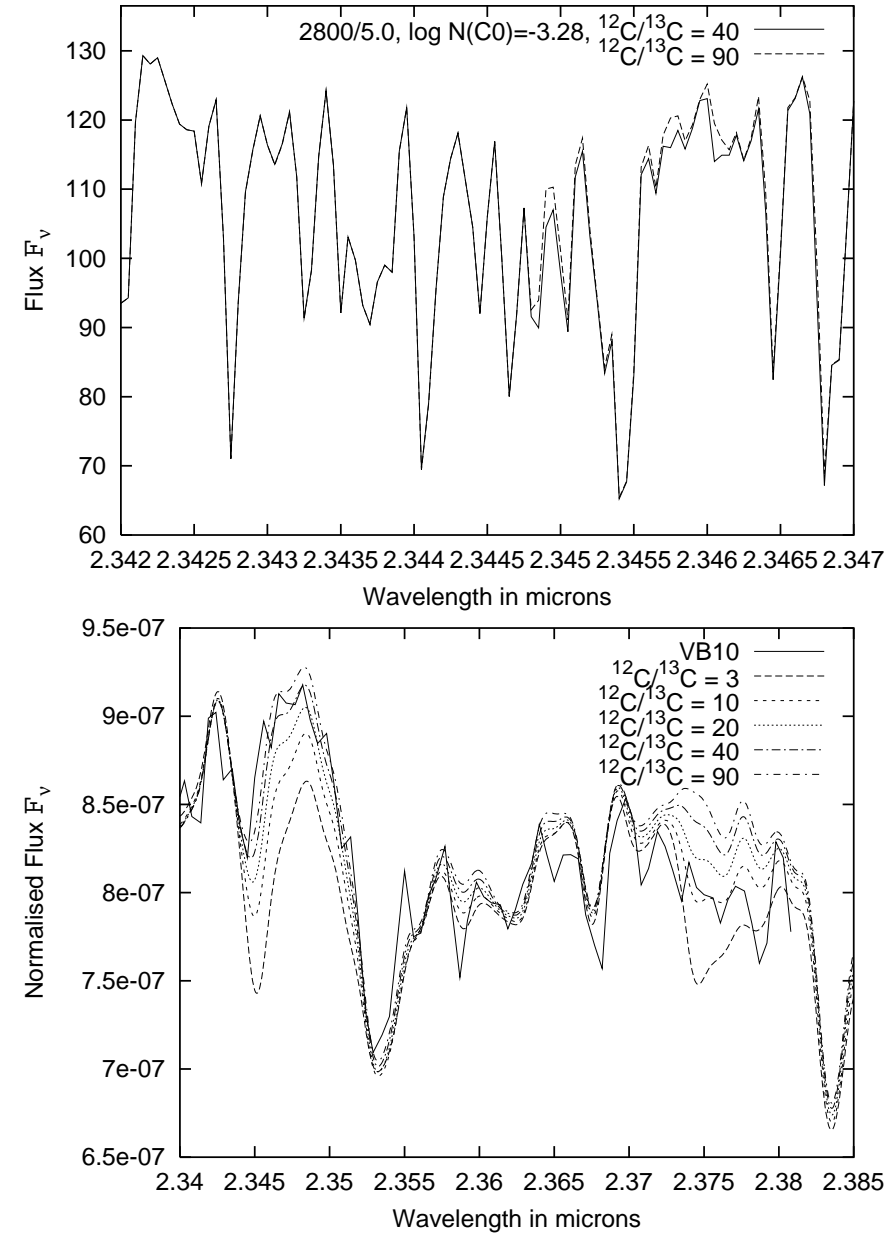

Fig. 9. Top: spectra computed for model atmosphere 2800/5.0, $\log N(\mathrm{C})=-3.28$, for ${ }^{12} \mathrm{C} /{ }^{13} \mathrm{C}=3$ to 90 at a resolution of around 46000 . Bottom: fits of computed spectra with different ${ }^{12} \mathrm{C} /{ }^{13} \mathrm{C}$ to VB10 observed spectrum.

The temperature structure of $\mathrm{M}$ dwarf atmospheres is smoother, in comparison to the giants. Indeed, their structure is governed by convection, which "smoothes" the temperature contrast between photosphere and upper layers, which are more pronounced in atmospheres of red giants. Atmospheres of cool dwarfs are denser than giants which strengthens the thermalisation of the radiation field. All these factors should reduce NLTE effects in CO lines formed in M dwarf atmospheres. More precise estimations are possible and would be desirable when making comparisons with higher resolution data though we consider to be beyond the scope of the current work.

Our computations were carried out for model atmospheres of solar abundance. Such abundances should be prevalent for the majority M dwarfs in the vicinity of solar system. Although, our sample of $\mathrm{M}$ dwarfs is rather small, we have found some deviation in carbon abundances from the solar case. A more refined solution should be made using self-consistent abundances of oxygen and nitrogen.

The model atmospheres used in this work were computed in the framework of the classical assumptions by Hauschildt et al. (1999). Energy transfer in atmospheres is provided by radiation field and convection. The reliability of the computed 

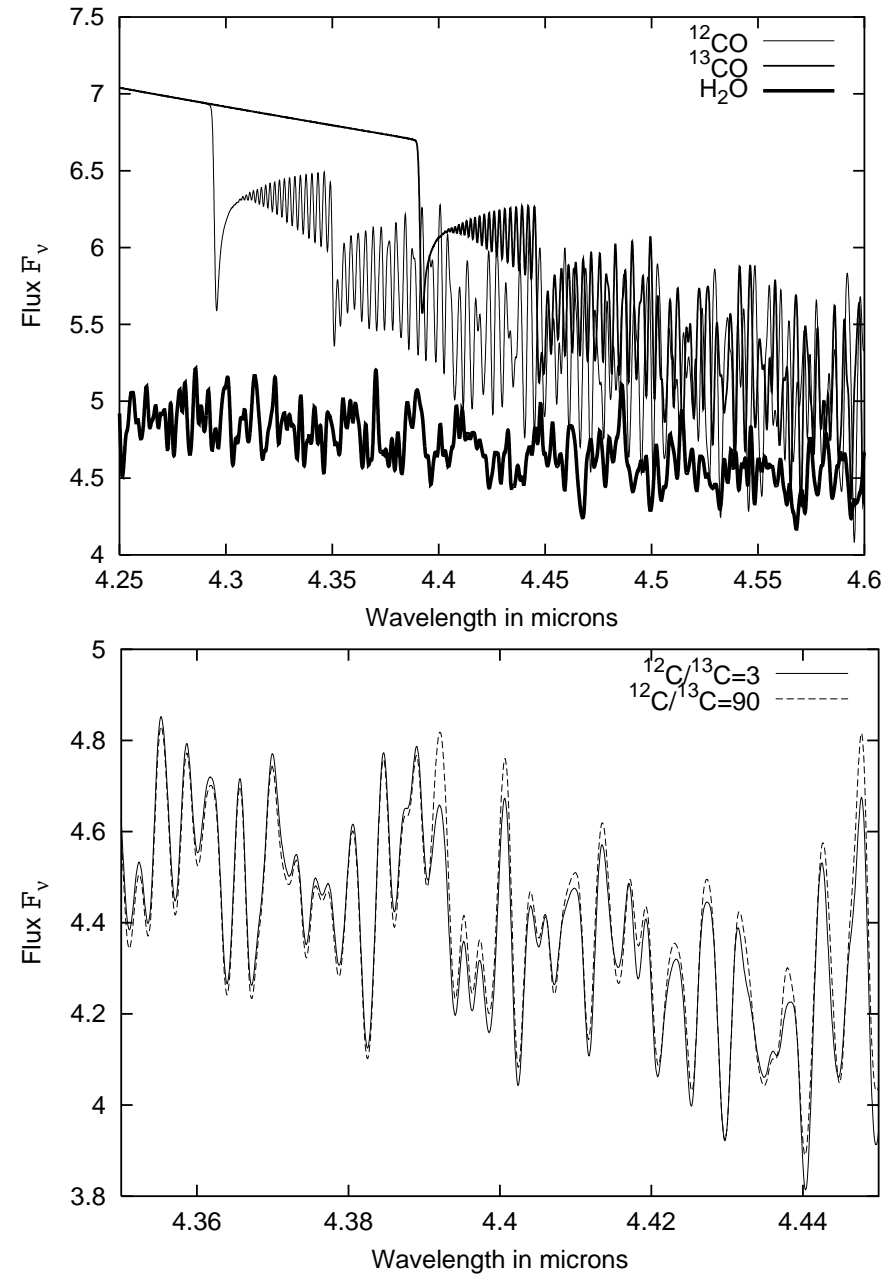

Fig. 10. Top: relative strength of ${ }^{12} \mathrm{C}^{16} \mathrm{O}, \mathrm{H}_{2} \mathrm{O}$ and ${ }^{13} \mathrm{C}^{16} \mathrm{O}$ bands in theoretical spectrum of M-dwarf 2800/5.0/0. and a combined spectra computed for the case of ${ }^{12} \mathrm{C}^{16} \mathrm{O}+\mathrm{H}_{2} \mathrm{O}$ and ${ }^{12} \mathrm{C}^{16} \mathrm{O}+{ }^{13} \mathrm{C}^{16} \mathrm{O}+$ $\mathrm{H}_{2} \mathrm{O}$ absorption. Bottom: synthetical spectra computed for $2800 / 5.0$ model atmospheres and different ${ }^{12} \mathrm{C} /{ }^{13} \mathrm{C}$. The resolution $R=4600$ was adopted.

model atmosphere is determined by both the completeness of the opacity sources as well as the model of convective processes (Ludwig et al. 1992). Absorption by $\mathrm{H}_{2} \mathrm{O}$ bands determines the opacity in the infrared spectra of red stars. However, the existing line lists are not sufficiently complete (e.g., Jones et al. 2002). The CO line list used in this work was computed for solar work and so is believed to be more than adequate for the lower energy states accessed in $\mathrm{M}$ dwarf atmospheres (Goorvitch 1994). Overall we do not consider that incompleteness in the $\mathrm{H}_{2} \mathrm{O}$ line lists will cause temperatures derived from $\mathrm{CO}$ bands to change by more than $150 \mathrm{~K}$.

The spectra presented here can not be used to determine reliable ${ }^{12} \mathrm{C} /{ }^{13} \mathrm{C}$ ratios. Nonetheless we propose that with a slightly different wavelength coverage than observed for this study, the ${ }^{13} \mathrm{CO}$ feature at $2.375 \mu \mathrm{m}$ should be a strong constraint on the ${ }^{12} \mathrm{C} /{ }^{13} \mathrm{C}$ ratio. For the other ${ }^{13} \mathrm{CO}$ feature we investigated the situation is complicated by ${ }^{13} \mathrm{C}^{16} \mathrm{O}$ bands forming amongst strong $\mathrm{H}_{2} \mathrm{O}$ absorption. One way of dealing with such "contamination" would be to obtain higher resolution data. In the upper panel of Fig. 9 we show synthetic spectra at a resolution of around 46000 , here even at low ${ }^{12} \mathrm{C} /{ }^{13} \mathrm{C}$ ratios the ${ }^{12} \mathrm{CO}$ and ${ }^{13} \mathrm{CO}$ bands can be distinguished. We have also investigated the possible use of the 4.4-4.6 micron region. However, this region does not appear to improve the possibility of determining ${ }^{12} \mathrm{C} /{ }^{13} \mathrm{C}$.

Acknowledgements. This work is based on observations obtained at the United Kingdom Infrared Telescope (UKIRT), which is operated by the Joint Astronomy Centre on behalf of the UK Particle Physics and Astronomy Research Council. We thank David Schwenke and David Goorvitch for providing AMES $\mathrm{H}_{2} \mathrm{O}$ and $\mathrm{CO}$ databases in digitial form. We are also grateful to the anonymous referee for a number of useful suggestions which significantly improved this manuscript. This work was partially supported by a PPARC visitors grant to the Astrophysics Research Institute, Liverpool John Moores University. YPs studies are partially supported by a Small Research Grant from American Astronomical Society.

\section{References}

Allard, F., \& Hauschildt, P. 1995, ApJ, 445, 433

Allard, F., Hauschildt, P. H., \& Schwenke, D. 2000, ApJ, 540, 1005

Aller, L. H., \& McLaughlin, D. B. 1965, Stellar structure (The University Chigago Press, Chicago and London), 1

Baldwin, J. R., Frogel, J. A., \& Persson, S. E. 1973, ApJ, 184, 427

Carbon, D. F., Milkey, R. W., \& Heasley, J. N. 1976, ApJ, 207, 253

Davidge, T. J., \& Boeshaar, P. C. 1993, ApJ, 403, 47L

Forbes, F. F., Stonaker, W. F., \& Johnson, H. L. 1970, AJ, 75, 158

Gerardy, C. L., Fesen, R. A., Hiflich, P., \& Wheeler, J. C. 2000, AJ, 119,2968

Goorvitch, D. 1994, ApJS, 95, 535

Gurvitz, L. V., Weitz, I. V., \& Medvedev, V. A. 1982, Thermodynamic properties of individual substances (Moscow Science)

Hauschildt, P., Allard, F., \& Baron, E. 1999, ApJ, 512, 377

Hinkle, K. H. 1978, ApJ, 220, 210

Heasley, J. N., \& Milkey, R. W. 1976, ApJ, 205, L43

Heasley, J. N., Ridgway, S. T., Carbon, D. F., Milkey, R. W., \& Hall, D. N. B. 1978, ApJ, 219, 970

Jones, H. R. A., Longmore, A. J., Jameson, R. F., \& Mountain, C. M. 1994, MNRAS, 267, 413

Jones, H. R. A., Longmore, A., Allard, F., \& Hauschildt, P. 1996, MNRAS, 280, 77

Jones, H. R. A., \& Tsuji, T. 1997, ApJ, 480, 39L

Jones, H. R. A., Pavlenko, Ya. V., Viti, S., \& Tennyson, J. 2002, MNRAS, 329, 290

Kraus, M., Krugel, E., Thum, C., \& Geballe, T. R. 2000, A\&A, 362, 158

Martin, E. 1999, MNRAS, 302, 59

Kirkpatrick, J. D., Henry, T. J., \& McCarthy, D. W. 1991, ApJS, 77, 417

Kirkpatrick, J. D., Kelly, D. M., Rieke, G. H., et al. 1993. ApJ, 402, 643

Kleinmann, S. G., \& Hall, D. N. B. 1986, ApJS, 62, 501

Kupka, F., Piskunov, N., Ryabchikova, T. A., Stempels, H. C., \& Weiss, W. W. 1999, A\&AS, 138, 119

Lazaro, C., Lynas-Gray, A. E., Clegg, R. E. S., Mountain, M., \& Zadrowny, A. 1991, MNRAS, 62

Leggett, S. K. 1992. ApJS, 82, 351

Leggett, S .K., Allard, F., Dahn, C., et al. 2000, ApJ, 535, 965

Leggett, S. K., Allard, F., Geballe, T. R., Hauschildt, P. H., \& Schweitzer, A. 2001, ApJ, 548, 908 
Laughlin, G., Bodenheimer, P., \& Adams, F. C. 1997, ApJ, 482, 420 Ludwig, H.-G., Allard, F., \& Hauschildt, P. H. 2002, A\&A, 395, 99 McGregor, P. J. 1987, ApJ, 312, 195

Palla, F., Bachiller, R., Stanghellini, L., Tosi, M., \& Galli, D. 2000, A\&A, 355, 69

Partrige, H., Schwenke, D. J. 1997, Chem. Phys., 106, 4618

Pavlenko, Ya. V. 2000, Astron. Rept., 44, 219

Pavlenko, Ya. V. 2002, Astron. Rept., 46, 567

Pilachowski, C., Sneden, C., Hinkle, K., \& Joyce, R. 1997, Metal-Poor Giants. Astron. J., 114, 819

Scott, A. D., Rawlings, J. M. C., Krautter, J., \& Evans, A. 1994, MNRAS, 268, 749

Schild, H., Boyle, S. J., \& Schmid, H. M. 1992, MNRAS, 258, 95
Schweitzer, A., Hauschildt, P. H., Allard, F., \& Basri, G. 1996, MNRAS, 283, 821

Sneden, C., \& Pilachowski, C. A. 1984, PASP, 96, 38

Suntzeff, N. B., \& Smith, V. V. 1991, ApJ, 381, 160

Tsuji, T. 1973, A\&A, 23, 411

Tsuji, T., Ohnaka, K., \& Aoki, W. 1996, A\&A, 305, 1

Tsuji, T., Ohnaka, K., \& Aoki, W. 1997, ASP Conf. Ser., 124, ed. H. Okuda, T. Matsumoto, \& T. Rollig, 91

Tsuji, T. 2002, ApJ, 575, 264

Viti, S., Jones, H., Maxted, P., \& Tennyson, J. 2002, MNRAS, 329, 290

Unsold, A. 1955, Physik der Sternatmospheren, 2nd ed. (Springer, Berlin) 